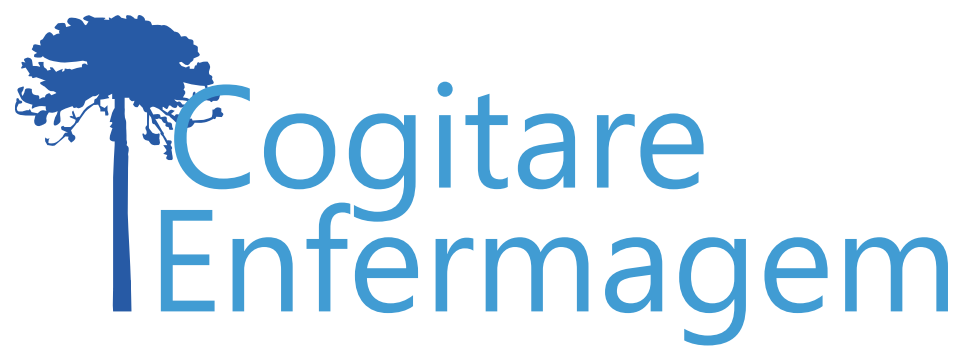

\title{
AUTOCUIDADO EM INDIVÍDUOS COM HANSENÍASE: AVALIAÇÃO DE PRÁTICAS NA REDE DE ATENÇÃO SECUNDÁRIA À SAÚDE*
}

\author{
Matheus de Medeiros Nóbrega1, Karen Krystine Gonçalves de Brito², Ester Missias Villaverde \\ Antas $^{3}$, Paula Soares Carvalho ${ }^{4}$, Emanuelle Malzac Freire de Santana ${ }^{5}$, Mirian Alves da Silva 6 , \\ Maria Júlia Guimarães Oliveira Soares ${ }^{7}$
}

\section{RESUMO}

Objetivo: avaliar as práticas de autocuidado com face, mãos e pés de indivíduos com hanseníase atendidos na rede de atenção secundária à saúde.

Método: estudo quantitativo, descritivo-exploratório, transversal, realizado em hospital de referência. População de 127 pessoas e amostra com 74. Coleta de dados realizada por meio de dois instrumentos estruturados e analisados pelos testes de Spearman e qui-quadrado.

Resultados: as práticas de autocuidado para os pés se mostraram melhor executadas em comparação às demais dimensões. Houve correlação significante nas práticas para mãos/pés e face/mãos. As associações foram significantes para classificação operacional/práticas para pés, comprometimento facial/práticas para face e comprometimento de membros inferiores/práticas para pés.

Conclusão: o estudo atente ao objetivo proposto e conclui que as práticas de autocuidado precisam ser melhor conhecidas e aplicadas, uma vez que tem sua eficácia reconhecida no meio científico, tratando-se de uma estratégia baseada em evidências.

DESCRITORES: Enfermagem; Atenção Secundária à Saúde; Educação em Saúde; Mycobacterium leprae; Promoção da Saúde.

*Artigo extraído da tese de doutorado "Adesão ao Autocuidado na Hanseníase à luz da Teoria de Everett Rogers". Universidade Federal da Paraíba, 2018.

COMO REFERENCIAR ESTE ARTIGO:

Nóbrega M de M, Brito K K G de, Antas EMV, Carvalho OS, Santana EMF de, Silva MA da, et al. Autocuidado em indivíduos com hanseníase: avaliação de práticas na rede de atenção secundária à saúde. Cogitare enferm. [Internet]. 2020 [acesso em "colocar data de acesso, dia, mês abreviado e ano"]; 25. Disponível em: http:// dx.doi.org/10.5380/ce.v25i0.65339.

\section{(c) (1)}

Este obra está licenciado com uma Licença Creative Commons Atribuição 4.0 Internacional.

${ }^{1}$ Enfermeiro. Mestrando em Enfermagem. Universidade Federal da Paraíba. João Pessoa, PB, Brasil. ()

${ }^{2}$ Enfermeira. Doutora em Enfermagem. Docente de Enfermagem da Faculdade Nova Esperança. João Pessoa, PB, Brasil. ${ }^{3}$ Enfermeira. Mestranda em Enfermagem. Universidade Federal da Paraíba. João Pessoa, PB, Brasil.

${ }^{4}$ Enfermeira. Mestranda em Enfermagem. Universidade Federal da Paraíba. João Pessoa, PB, Brasil. 0

${ }^{5}$ Fisioterapeuta. Doutoranda em Enfermagem. Docente da Faculdade Nova Esperança. João Pessoa, PB, Brasil. (

${ }^{6}$ Enfermeira. Doutora em Enfermagem. Docente de Enfermagem da Universidade Federal da Paraíba. João Pessoa, PB, Brasil. (인

${ }^{7}$ Enfermeira. Doutora em Enfermagem. Docente de Enfermagem da Universidade Federal da Paraíba. João Pessoa, PB, Brasil. 


\title{
SELF-CARE IN INDIVIDUALS WITH LEPROSY: EVALUATING PRACTICES IN THE SECONDARY HEALTH CARE NETWORK
}

\begin{abstract}
Objective: To evaluate self-care practices for face, hands and feet of individuals with leprosy treated in the secondary health care network.

Method: Quantitative, descriptive-exploratory, cross-sectional study carried out in a reference hospital. Population of 127 people and sample with 74. Data collection performed using two structured instruments and analyzed by the Spearman and chi-square tests.

Results: The self-care practices for the feet were better performed compared to the other dimensions. There was a significant correlation in the practices for hands/feet and face/ hands. The associations were significant for operational classification/practices for feet, facial impairment/practices for face and impairment of lower limbs/practices for feet.

Conclusion: The study is attentive to the proposed objective and concludes that self-care practices need to be better known and applied, since their effectiveness is recognized in the scientific community, as it is an evidence-based strategy.
\end{abstract}

DESCRIPTORS: Nursing; Secondary Health Care; Health education; Mycobacterium leprae; Health Promotion.

\section{AUTOCUIDADO EN SUJETOS CON HANSENÍASIS: EVALUACIÓN DE PRÁTICAS EM LA RED DE ATENCIONN SECUNDARIA DE SALUD}

\begin{abstract}
RESUMEN
Objetivo: evaluar las prácticas de autocuidado en el rostro, manos y pies de personas con lepra tratadas en la red de atención secundaria de salud.

Método: estudio cuantitativo, descriptivo-exploratorio, transversal, realizado en un hospital de referencia. Población de 127 personas y muestra de 74. Recolección de datos realizada con dos instrumentos estructurados y analizados por Spearman y pruebas de chi-cuadrado. Resultados: las prácticas de autocuidado de los pies se realizaron mejor si comparadas con las otras dimensiones. Hubo una correlación significativa en las prácticas con manos / pies y rostro / manos. Las asociaciones fueron significativas para la clasificación operativa / prácticas para los pies, deterioro facial / prácticas para el rostro y deterioro de extremidades inferiores / prácticas para los pies.

Conclusión: el estudio alcanza el objetivo propuesto y concluye que las prácticas de autocuidado deben conocerse y aplicarse más, una vez que se ha reconocido su eficacia en el medio científico, dado que se trata de una estrategia basada en evidencias.
\end{abstract}

DESCRIPTORES: Enfermería; Atención Secundaria de la salud; Educación en Salud; Mycobacterium leprae; Promoción de Salud. 
Discorrer sobre hanseníase é remeter-se a uma importante doença que aparentemente sempre existiu, dada a sua origem milenar, mas que até então se apresenta às comunidades nacionais e internacionais subdesenvolvidas como problema de saúde pública. Da Ásia à América do Sul, é vasta a amplitude de pessoas que são infectadas pelo Mycobacterium leprae, seu agente etiológico, e que desenvolvem a patologia em suas formas clínicas ${ }^{(1)}$.

Todavia, o impacto negativo mais significativo da hanseníase traduz-se nas incapacidades, que variam de formas discretas às graves deformidades que atingem principalmente a tríade "face, mãos e pés", em seu prognóstico mais agressivo, agregando malefícios como o medo e estigma na vida dos doentes ${ }^{(2)}$. Ademais, considerando que a hanseníase acomete pessoas em idade socioeconomicamente ativa, as sequelas comprometem também a participação dinâmica e funcional delas em seu cotidiano(3-5).

Na contramão das incapacidades físicas, a Organização Mundial de Saúde (OMS), bem como o Ministério da Saúde (MS) brasileiro, propõe a utilização de indicadores operacionais e epidemiológicos que consideram o número de pacientes avaliados e graus de incapacidade física (GIF) 1 e 2 para mensurar a qualidade da assistência em saúde. Enfatizam ainda a adoção de estratégias preventivas recomendadas por estudos de relevância, como o autocuidado, por meio de técnicas financeiramente acessíveis e de fácil realização(6-7).

O MS conceitua o autocuidado como "procedimentos, técnicas e exercícios que o próprio doente pode realizar no seu domicílio e em outros ambientes"(6). Entretanto, pioneiramente, o autocuidado é baseado na teoria de enfermagem de Orem, que centraliza o indivíduo como protagonista do seu próprio cuidado. Na impossibilidade de sua realização, o enfermeiro o auxilia, partindo da sua competência de orientar e ajudar. Além disso, o autocuidado possibilita a autopercepção, o empoderamento e o cuidado independente, assim como envolve vigilância e cuidado corporal ${ }^{(8-9)}$.

Contudo, a dinâmica do autocuidado não se restringe à prática e ao auxílio quando necessário. Envolve também a percepção do indivíduo com hanseníase sobre seu estado de saúde, suas limitações e potencialidades, seu desejo de prevenir-se contra as incapacidades, assim como para o profissional que o assiste, trazendo a responsabilidade de estar atento à conjuntura psicossocial do seu cliente sem separar o corpo da mente e de orientá-lo sempre que necessário(10).

Partindo desse contexto, é preciso fomentar a necessidade de se intervir desde a primeira consulta, utilizando-se do processo de educação em saúde para viabilizar as práticas de autocuidado, e dessa forma promover a saúde, prevenir agravos e reabilitar indivíduos. Tais ações devem ser incentivadas e orientadas, visto que são consideradas de simples execução e importantes para o processo terapêutico, dependente do conhecimento, harmonia e comunicação do cuidador e paciente ${ }^{(11-12)}$.

A atenção secundária à saúde composta por serviços ambulatoriais e hospitalar é contínua à atenção primária no modelo das redes de atenção à saúde, atuando na intervenção para casos mais graves de hanseníase, no tratamento de incapacidades e no apoio para a realização de práticas de autocuidado, sem negligenciar a prevenção e promoção da saúde individual(6,13).

Portanto, reconhece-se a importância de avaliar o comportamento nas práticas de autocuidado, posto que é fundamental para diminuir as consequências negativas da doença, proporcionando bem-estar e saúde. Então, esta pesquisa indaga: como estão sendo realizadas as práticas de autocuidado de indivíduos com hanseníase na face, mãos e pés?

Procurando respostas para esta questão, a investigação objetivou avaliar as práticas de autocuidado com face, mãos e pés de indivíduos com hanseníase atendidos na rede de 
atenção secundária à saúde.

\section{MÉTODO}

Trata-se de um estudo transversal, descritivo-exploratório, com abordagem quantitativa, tendo como cenário o ambulatório de dermatologia de um hospital de referência em hanseníase na Paraíba.

A população do estudo foi estabelecida a partir dos registros cadastrais do referido ambulatório, para o mês de janeiro de 2017 (ano em que a coleta de dados foi realizada), onde constataram-se 127 indivíduos em acompanhamento. Partindo deste dado, estabeleceram-se os critérios de inclusão: maiores de 18 anos de idade, uso do tratamento poliquimioterápico a partir da $2^{\mathrm{a}}$ dose. Foram excluídos indivíduos com cognição prejudicada autorreferida ou percebida.

O tamanho da amostra para se obter representatividade foi alcançado a partir do cálculo amostral de fórmula $\mathrm{n}=\left(\mathrm{N} \times \mathrm{n}_{0}\right) /\left(\mathrm{N}+\mathrm{n}_{0}\right)$, assim:

$\mathrm{n}^{0}$ - primeira aproximação para o tamanho da amostra (número de casos registrados no cenário da pesquisa $=127)$;

e - erro amostral tolerável=0,05;

N - tamanho da população (119 casos novos de hanseníase registrados na região metropolitana de João Pessoa/Paraíba, para o ano de $2016^{(14)}$.

$\mathrm{n}$ - tamanho da amostra $=62$ indivíduos.

Por se tratar de uma amostra probabilística de fluxo por conveniência, obteve-se o quantitativo de 74 sujeitos entrevistados, considerando o período delimitado para o estudo, de janeiro a março de 2017.

A coleta de dados foi realizada por meio de dois instrumentos estruturados, o primeiro contendo dados sociodemográficos (sexo, idade, nível de escolaridade, estado civil, renda familiar média, religião), e clínico-epidemiológicos (dose do tratamento, classificação operacional, status de baciloscopia, forma clínica, grau de incapacidade física no diagnóstico, comprometimento neural).

O segundo instrumento utilizado é constituído por 46 questões relacionadas ao autocuidado de pacientes com hanseníase, sendo divididas em três dimensões: 13 questões para face, 11 para mãos e 22 para pés. Cada questionamento do instrumento admite uma resposta, cuja pontuação pode variar de 1 a 5 . Assim, entende-se que 1 corresponde a "Nunca", 2 "uma a duas vezes na semana", 3 "não sei/frequência indefinida", 4 "três a quatro vezes na semana" e 5 a "Sempre". Entretanto, algumas questões possuem conotação negativa, invertendo em sentido decrescente o score de classificação da resposta. Quanto às dimensões, estas admitem diferentes scores parciais, a saber: 13 - 65 pontos para face, 11 - 55 pontos para mãos e 22 - 110 pontos para pés. Para avaliação das práticas de autocuidado, calculou-se a média aritmética da pontuação de cada dimensão, classificando as práticas de autocuidado dos sujeitos em "insatisfatórias" (média de 1,0 2,5 pontos), "precisa melhorar" (média de 2,6 - 3,5 pontos) e "satisfatórias" (média de 3,6 $-5,0$ pontos).

Ressalta-se que o instrumento foi construído como parte da tese de doutoramento de um componente do Grupo de Estudos e Pesquisa em Feridas da Universidade Federal da Paraíba, sendo posteriormente submetido ao processo de validação.

Os dados foram analisados descritiva e inferencialmente pelo teste de Spearman 
para medir a correlação de classificação das dimensões como variáveis ordinais, e Chiquadrado pelo teste de Friedman para determinar a associação entre variáveis clínicas e práticas de autocuidado. Considerou-se significância estatístiça p-valor $\leq 0,05$.

O projeto foi aprovado pelo Comitê de Ética e Pesquisa do Centro de Ciências em Saúde da Universidade Federal da Paraíba, sob o protocolo n0785/16 e de acordo com o Certificado de Apresentação para Apreciação Ética de número 62360816.3.0000.5188. Foram obedecidas todas as observâncias éticas contempladas na Resolução n 466/12 do Conselho Nacional de Saúde(15).

\section{RESULTADOS}

Obteve-se amostra representativa de 74 sujeitos entrevistados, com predominância sociodemográfica de homens 58,1\%; idade entre 18 e 82 anos (média 43,4; DP \pm 15,09); estado civil casado ou em união estável 51,4\%; nível de escolaridade ensino fundamental $59,5 \%$; renda familiar menor ou igual a um salário mínimo 56,8\%; religião católica $62,2 \%$; indivíduos pardos 50,0\%; e residência própria 67,6\%.

Com relação às variáveis clínico-epidemiológicas, predominou multibacilares 86,5\%; baciloscopia positiva 55,4\%; forma clínica dimorfa 47,3\%; GIF diagnóstico zero 52,7\%; sem comprometimento facial $93,2 \%$ e de membros superiores (MMSS) 68,9\%; com comprometimento de membros inferiores (MMII) 48,6\%.

Endereçando o objetivo deste estudo, as classificações das práticas de autocuidado na face, mãos e pés são descritas na Tabela 1.

Tabela 1 - Distribuição da classificação das práticas de autocuidado nas três dimensões em suas frequências absolutas e percentuais. João Pessoa, PB, Brasil, 2018

\begin{tabular}{lcccccc} 
Classificação & \multicolumn{2}{c}{ Face } & \multicolumn{2}{c}{ Mãos } & \multicolumn{2}{c}{ Pés } \\
\cline { 2 - 8 } & $\mathbf{n}$ & $\%$ & $\mathbf{n}$ & $\%$ & $\mathbf{n}$ & $\%$ \\
\hline Satisfatória & 1 & 1,4 & 8 & 10,8 & 17 & 23 \\
\hline Precisa melhorar & 54 & 73 & 44 & 59,5 & 57 & 77 \\
\hline Insatisfatória & 19 & 25,6 & 22 & 29,7 & - & -
\end{tabular}

Considerando a classificação satisfatória, esses resultados mostram que as práticas de autocuidado para os pés são melhor executadas em comparação às demais, seguida das práticas para mãos e por último para a face.

Para compreender a correlação entre as variáveis do estudo, a Tabela 2 mostra os coeficientes de correlação entre as dimensões e seus índices de significância. 
Tabela 2 - Distribuição da correlação das dimensões através do teste de Spearman com seus coeficientes de correlação e Valores-p. João Pessoa, PB, Brasil, 2018

\begin{tabular}{lcc} 
Correlações & Coeficiente de Correlação* & Valor-p \\
\hline Face e Mãos & 0,25 & $0,032^{\star \star}$ \\
\hline Face e Pés & 0,21 & 0,073 \\
\hline Mãos e Pés & 0,27 & $0,020^{\star \star}$ \\
*Teste de Correlação de Spearman. **Valores estatisticamente significativos $(p<0,05)$.
\end{tabular}

Esses resultados traduzem a fraca, porém significativa correlação diretamente proporcional entre a dimensões face-mãos e mãos-pés.

Para verificar a associação de variáveis intervenientes ao processo de agravamento do quadro clínico na hanseníase, foi realizada inferência de associação estatística entre aquelas e as práticas de autocuidado, conforme apresentado na Tabela 3.

Tabela 3 - Distribuição do teste de associação entre variáveis pelo teste de Friedman, com seus valores de Chi-quadrado e Valores-p. João Pessoa, PB, Brasil, 2018

\begin{tabular}{lcc} 
Associações & Valor Qui-Quadrado* & Valor-p \\
\hline Classificação Operacional x Práticas da Face & 3,240 & 0,072 \\
\hline Classificação Operacional x Prática das Mãos & 1,059 & 0,303 \\
\hline Classificação Operacional x Prática dos Pés & 23,000 & 0,01 ** \\
\hline Comprometimento Facial x Práticas da Face & 14,727 & $0,01^{* *}$ \\
\hline Comprometimento de MMSS x Prática das Mãos & 0,000 & 1,000 \\
\hline Comprometimento de MMII x Prática dos Pés & 31,837 & 0,01 ** \\
*Teste de Associação de Friedman. **Valores estatisticamente significativos $(p<0,05)$. &
\end{tabular}

\section{DISCUSSÃO}

Estudos epidemiológicos recentemente publicados na literatura descrevem amostras com características sociodemográficas e clínicas semelhantes a este estudo, como sexo, raça, nível de escolaridade, forma clínica e classificação operacional corroborando as variáveis descritas e mostrando o perfil epidemiológico predominante na atualidade ${ }^{(16-18)}$.

Considerando as práticas de autocuidado, os indivíduos avaliados no grupo "Precisa melhorar", embora apresentem falhas quanto à frequência de execução, ainda são considerados parcialmente positivos, posto que executam de forma moderada (média de $2,6-3,5$ pontos, ou seja, corresponde a uma média de três vezes por semana). Práticas classificadas como "Insatisfatórias" possuem aspecto negativo para os sujeitos, ao passo em que são realizadas com frequência esporádica, ou mesmo não são realizadas. Para esse 
último grupo, é necessária a identificação preliminar de problemas inerentes à realização ou não do autocuidado e de intervenções que o efetivem e incentivem. Esse processo pode ser orientado mediante educação em saúde conduzida pelos profissionais da área e pela compreensão e iniciativa dos sujeitos ${ }^{(19)}$.

A autopercepção, o conhecimento sobre a gravidade da doença e os possíveis efeitos sobre seu corpo, o empoderamento pessoal e a confiança em suas potencialidades são fatores determinantes para a formação do alicerce e pilares de sustentação emocional e motivacional, para então abraçar as práticas regulares do autocuidado, em prol de sua saúde e bem-estar ${ }^{(8,10)}$.

Outras estratégias podem fortalecer tanto o vínculo profissional-cliente, como a integração das ações de autocuidado. Os grupos de autocuidado podem satisfazer essas necessidades relacionadas à educação em saúde para indivíduos com hanseníase ${ }^{(8)}$. Tal método proporciona aos participantes a oportunidade de trocar mutuamente conhecimento, contar experiências e saberes, e aprender com outros sobre os perigos incapacitantes da hanseníase e como preveni-los através do autocuidado.

Para além da análise isolada de suas práticas diárias, e considerando o perfil clínico dos indivíduos, observa-se que o comprometimento dos membros inferiores $(48,6 \%)$ foi - maior entre as três dimensões, convergindo com a classificação "Precisa melhorar" predominante, consequência da realização parcialmente satisfatória da prática de autocuidado. Algumas ideias podem atuar como hipóteses nesse caso, como a consciência de risco desenvolvida a partir do comprometimento já instalado que afetam a sensibilidade e locomoção, despertando nos sujeitos a necessidade do autocuidado para o controle de agravos.

Sobre a dimensão dos MMII, estudo asiático que abordou o autocuidado em úlceras plantares traz resultados que denotam negatividade nesse tipo de cuidado(20). Seguindo os achados, estudo brasileiro sobre as práticas de autocuidado mostra resultados que refletem o cuidado deficiente dos indivíduos com hanseníase para com os pés ${ }^{(21)}$. Ambos corroboram os resultados desse estudo, ao passo em que, embora se observe melhor desempenho das ações com os MMII, a maioria dos participantes ainda aponta percentuais distantes do desejado - Prática satisfatória (23\%).

Mãos e face apresentaram índices insatisfatórios de execução de práticas comparados aos pés. $O$ índice baixo de comprometimento facial $(6,8 \%)$ para face junto às suas práticas insatisfatórias $(25,6 \%)$ reforça a ideia de que não está sendo realizado o autocuidado na tentativa de prevenir as incapacidades, pois entende-se que o fato de não apresentar acometimento possa estar sendo interpretado como desnecessária a realização do cuidado. Além disso, durante a coleta de dados, os sujeitos expressavam não ter conhecimento sobre práticas para a face.

Processo similar pode ser evidenciado em relação à dimensão mãos, em que também houve menor frequência de comprometimento dos MMSS $(31,1 \%)$ e maior classificação de práticas insatisfatórias $(29,7 \%)$. Neste caso, no entanto, a preocupação é maior, dado que comparativamente à dimensão face, o percentual de indivíduos acometidos é ampliado.

Dessa maneira, as evidências indicam que a consciência de risco a partir do déficit de conhecimento está afetada, levando às fragilidades das práticas de prevenção de incapacidades para mãos e face. Estudo aponta a baixa percepção do risco hansênico que reflete a deficiência no autocuidado(22). Tal resultado torna-se uma ameaça para a integridade física e psicológica e para a imagem pessoal, ainda mais considerando que as incapacidades podem aparecer em qualquer fase da doença, necessitando da realização do autocuidado o quanto antes ${ }^{(23-24)}$.

Ressalta-se, em complementariedade às informações acima, que a caracterização clínica dos indivíduos representa majoritariamente classificação multibacilares e dimorfa na amostra. Os axiomas entre esses resultados apresentam uma conjuntura em que o indivíduo acometido apresenta a forma grave da doença, com alto potencial de acometimento neural 
e, portanto, mais propício ao desenvolvimento de deformidades e incapacidades. Ainda neste contexto, a forma dimorfa se apresenta patologicamente por sua diversidade de sinais e sintomas, sendo considerada de forte magnitude pela instabilidade imunopatológica.

No tocante às correlações estatísticas, observa-se que os sujeitos tratam o autocuidado da face $x$ mãos e de mãos $x$ pés como conjuntos ou similares. Estudos atuais em incapacidades na hanseníase registram predominância precoce de comprometimento nos membros inferiores em suas amostras ${ }^{(25-26)}$. Dessa forma, pode haver a eventualidade das dimensões face e mãos serem consideradas separadas da dimensão pés, pelo fato de os sujeitos não associarem os agravos entre as dimensões.

Por outro lado, a fraca correlação entre os MMSS e MMII parece estar relacionada a execuções simultâneas e uso de componentes adjuvantes (hidratantes, lubrificantes, lixas d'água, entre outros) semelhantes, conforme orientações do manual do MS que trata sobre as incapacidades e autocuidado ${ }^{(27)}$.

Acredita-se também que alguns desses cuidados são realizados corriqueiramente, independentes do acometimento patológico. Além disso, o presente estudo apresenta os maiores índices de prática satisfatória de mãos e pés, ainda que minguado, ao contrário para face, corroborando também a correlação investigada. Estudo qualitativo auxilia o fortalecimento da ideia de que as dimensões mãos e pés convergem nas suas representações e interpretações para a imagem corporal do indivíduo com hanseníase, principalmente para aqueles com incapacidades instaladas ${ }^{(22)}$.

Além de correlacionar as dimensões, é interessante testar associações entre variáveis sociodemográficas e clínicas e as práticas de autocuidado nas três dimensões, pois compreende-se que essas podem influenciar na performance das atividades do autocuidado e expressar valiosos significados.

As associações entre classificação operacional e o comprometimento de membros inferiores convergem para as práticas de autocuidado nos pés. Em outras palavras, a multibacilaridade predominante na amostra é caracterizada não só pelo maior número de lesões corporais, mas também pela maior carga bacilar no organismo (2). Consequentemente, o comprometimento de membros torna-se ainda maior nessa relação diretamente proporcional, devido aos danos neurais, facilitando assim a incapacitação física, dado similar a outro estudo(28).

Posto isso, e considerando a associação estatística entre práticas do autocuidado em pés e comprometimento destes juntamente à multibacilaridade, pode-se inferir que a consciência sobre o risco de agravos da doença leva à significante tendência da execução do autocuidado pelos sujeitos, seja ela satisfatória ou parcialmente satisfatória ${ }^{(10)}$.

Quanto ao comprometimento facial e práticas de autocuidado, as variáveis apresentam associação dependente, ou seja, a amostra entende que para que haja práticas é preciso o acometimento. Como não há a presença considerável de acometimento facial na amostra, as práticas satisfatórias foram mínimas $(1,4 \%)$, assim, entende-se que caso os sujeitos não apresentem sequelas faciais, não se cuidam por prevenção.

As incapacidades faciais são notáveis e podem afetar a autoestima e sua apresentação social, além da qualidade do sono e da visão. Além disso, podem se manifestar na forma de lagoftalmo, triquíase, entre outras, além de alterações na morfologia nasal e nos músculos faciais. Portanto, a hanseníase traz consigo toda a carga negativa de doença que incapacita, deforma e que pode deixar sequelas, sobretudo para a face, que é considerada a identidade e apresentação pessoal(29).

É válido lembrar que o desenvolvimento de incapacidades físicas pode, além de medir a magnitude da patologia, ser um indicativo de que o diagnóstico provavelmente foi tardio ou que a forma clínica foi definida inadequadamente ${ }^{(30)}$.

Infelizmente, são muitas as questões que levam as pessoas com hanseníase a não 
realizarem o autocuidado de forma e frequência correta. Observou-se durante as coletas que isso se dá pela deficiência de conhecimento sobre os riscos que a hanseníase pode acarretar, ou pela falta de tempo e recursos materiais, pelo pouco estímulo familiar, social ou mesmo dos profissionais de saúde.

Portanto, vê-se a necessidade do reforço de sua importância, da abertura de propostas para intervenções educativas em autocuidado, e do entendimento do ser, suas limitações, suas vontades e percepções, elementos que podem influenciar na adesão ao autocuidado e sua prática efetiva.

Por outro lado, para as práticas que precisam melhorar, é necessária a vigilância, a reafirmação da responsabilidade do autocuidado e a educação em saúde, assim como para as práticas já satisfatórias para que continuem sendo classificadas nessa qualidade. Nesse contexto, este estudo traz consigo a descrição de algumas nuances que podem ser constatadas dentro dessa temática que envolve a dependência de circunstâncias, condições e outras questões pertinentes ao indivíduo com hanseníase.

O estudo apresentou limitações concernentes ao entendimento íntimo e aprofundado sobre os sujeitos e suas peculiaridades frente à execução de práticas do autocuidado e ao que pode influenciá-las. Ressalta-se em especial a oportunidade de novos estudos qualitativos, a fim de preencher lacunas aqui encontradas, de responder e reformular hipóteses.

\section{CONCLUSÃO}

A pesquisa aponta descrição sociodemográfica e clínica de sujeitos acometidos pela hanseníase similar à literatura científica posta. Ao analisar as práticas de autocuidado com as dimensões face, mãos e pés, observou-se melhor resposta dos entrevistados para as ações direcionadas aos membros inferiores, seguido pelos superiores e, por fim, pela face. Ainda assim, os resultados apontam que as práticas não apresentam a qualidade e/ou frequências necessárias para serem consideradas satisfatórias.

Aquém à regularidade de realização do autocuidado, os dados apontaram correlação e associações entre estas e a presença de comprometimento com a dimensão analisada (à exceção dos MMSS), de forma que parece ser necessária a percepção do acometimento da face ou pés para que as ações de autocuidado sejam realizadas.

Diante do exposto, o estudo atente ao objetivo proposto, e, portanto, conclui-se que as práticas de autocuidado precisam ser melhor conhecidas e aplicadas, uma vez que têm sua eficácia reconhecida no meio científico, tratando-se de uma estratégia baseada em evidências. Para além do conhecimento, é preciso o incentivo à sua adesão e a investigação de fatores que podem tornar-se intervenientes ao processo de adoção pelos indivíduos.

\section{REFERÊNCIAS}

1. Noriega LF, Noriega AF, Vieira ML, Chiacchio ND, Pereira GAAM. Leprosy: ancient disease remains a public health problem nowadays. An Bras Dermatol [Internet]. 2016 [acesso em 15 jan 2018]; 91(4). Disponível em: http://dx.doi.org/10.1590/abd1806-4841.20164861.

2. Fischer M. Leprosy - an overview of clinical features, diagnosis, and treatment. J Dtsch Dermatol Ges [Internet]. 2017 [acesso em 15 jan 2018]; 15(8). Disponível em: https://doi.org/10.1111/ddg.13301.

3. Monteiro LD, Alencar CH, Barbosa JC, Novaes CCBS, Silva R de CP, Heukelbach J. Limited activity and 
social participation after hospital discharge from leprosy treatment in a hyperendemic area in north Brazil. Rev. bras. epidemiol. [Internet]. 2014 [acesso em 15 jan 2018]; 17(1):91-104. Disponível em: http://www. scielo.br/pdf/rbepid/v17n1/pt 1415-790X-rbepid-17-01-00091.pdf.

4. Queirós MI, Alencar CHM, Sena AL, Ramos Júnior AN, Monteiro LD, Barbosa JC. Clinical and epidemiological profile of leprosy patients attended at Ceará, 2007-2011. An. Bras. Dermatol. [Internet]. 2016 [acesso em 15 jan 2018]; 91(3). Disponível em: http://dx.doi.org/10.1590/abd1806-4841.20164102.

5. Valentin DC, Candelario N, Carrasquillo OY, Figueroa L, Sanchéz JL. Leprosy in Puerto Rico: insight into the new millennia. Int. J. Dermatol. [Internet]. 2017 [acesso em 15 jan 2018]; 56(4). Disponível em: https:// doi.org/10.1111/ijd.13496.

6. Ministério da Saúde (BR), Secretaria de Vigilância em Saúde. Departamento de Vigilância das Doenças Transmissíveis. Diretrizes para vigilância, atenção e eliminação da hanseníase como problema de saúde pública: manual técnico-operacional. Brasília: Ministério da Saúde; 2016.

7. Marega A, Pires P das N, Mucufo J, Muloliwa A. Hansen's disease deformities in a high risk area in Mozambique: A case study. Rev Soc Bras Med Trop [Internet]. 2019 [acesso em 15 maio 2019]; 52:e20180103. Disponível em: http://dx.doi.org/10.1590/0037-8682-0103-2018.

8. Pinheiro MGC, Silva SYB e, Silva F de S, Ataide CAV, Lima IB de, Simpson CA. Knowledge on prevention of disabilities in a hanseniasis self-care group. REME Rev Min Enferm [Internet]. 2014 [acesso em 15 jan 2018]; 18(4). Disponível em: http://www.dx.doi.org/10.5935/1415-2762.20140066.

9. White C, Franco-Paredes C. Leprosy in the 21st Century. Clin Microbiol Rev [Internet]. 2015 [acesso em 15 jan 2018]; 28(1). Disponível em: http://doi.org/10.1128/CMR.00079-13.

10. Souza IA, Ayres JA, Meneguin S, Spagnolo SA. Autocuidado na percepção de pessoas com hanseníase sob a ótica da complexidade. Esc. Anna Nery [Internet]. 2014 [acesso em 15 jan 2018]; 18(3). Disponível em: http://dx.doi.org/10.5935/1414-8145.20140072.

11. Duarte MTC, Ayres JA, Simonetti JP. Consulta de enfermagem: estratégia de cuidado ao portador de hanseníase em atenção primária. Texto contexto - enferm [Internet]. 2009 [acesso em 24 abr 2018]; 18(1). Disponível em: http://dx.doi.org/10.1590/S0104-07072009000100012.

12. Monteiro BR, Pinheiro MGC, Isoldi DMR, Cabral AMF, Simpson CA, Mendes FRP. Hanseníase: enfocando a educação em saúde para o projovem. Rev. pesqui. cuid. fundam. [Internet]. 2015 [acesso em 06 fev 2018]; 7(5). Disponível em: http://dx.doi.org/10.9789/2175-5361.2015.v7i5.49-55.

13. Erdmann AL, Andrade SR de, Mello ALSF de, Drago LC. A atenção secundária em saúde: melhores práticas na rede de serviços. Rev Lat Am Enfermagem [Internet]. 2013 [acesso em 06 fev 2018]; 21 (nspe). Disponível em: http://www.scielo.br/pdf/rlae/v21nspe/pt 17.pdf.

14. Ministério da Saúde (BR), Departamento de Informática do SUS. Hanseníase: indicadores operacionais e epidemiológicos [Internet]. 2016 [acesso em 22 maio 2018]. Disponível em: http://tabnet.datasus.gov. $\mathrm{br} / \mathrm{cgi} /$ sinannet/hanseniase/hans indicadores.htm.

15. Conselho Nacional de Saúde (BR). Resolução 466, de 12 de Dezembro de 2012: dispõe sobre diretrizes e normas regulamentadoras de pesquisas envolvendo seres humanos [Internet]. 2012 [acesso em 06 fev 2018]. Disponível em: http://conselho.saude.gov.br/resolucoes/2012/reso466.pdf.

16. Nazario AP, Ferreira J, Faccini-Schuler L, Fiegebaum M, Artigalás O, Vianna FSL. Leprosy in Southern Brazil: a twenty-year epidemiological profile. Rev Soc Bras Med Trop [Internet]. 2017 Mar [acesso em 18 abr 2018];50(2). Disponível em: http://doi.org/10.1590/0037-8682-0229-2016.

17. Nobre ML, Illarramendi X, Dupnik KM, Hacker MA, Nery JAC, Jerônimo SMB. Multibacillary leprosy by population groups in Brazil: Lessons from an observational study. PloS Negl Trop Dis [Internet]. 2017 [acesso em 12 jun 2018];11(2). Disponível em: https://doi.org/10.1371/journal.pntd.0005364.

18. Campos MRM, Batista AVA, Guerreiro JV. Perfil clínico-epidemiológico dos pacientes diagnosticados com hanseníase na Paraíba e no Brasil, 2008- 2012. Rev. bras. ciênc. saúde [Internet]. 2018 [acesso em 18 
abr 2018]; 22(1). Disponível em: http://doi.org/10.4034/rbcs.2018.22.01.11.

19. D'Azevedo SSP, Freitas EN de, Nascimento LO, Santos DCM, Nascimento RD. Perception of patients with lepra about the self-care groups. J Nurs UFPE on line. 2018 [acesso em 19 abr 2018]; 12(6). Disponível em: https://periodicos.ufpe.br/revistas/revistaenfermagem/article/viewFile/230855/29199.

20. Win LL, Shwe S, Maw W, Ishida Y, Myint K, Mar KK, et al. Why person affected by leprosy did not look after their plantar ulcer? Experience from Pakokku zone, Myanmar. Jpn J Lepr [Internet]. 2010 [acesso em 24 out 2018]; 79(3). Disponível em: https://www.jstage.jst.go.jp/article/hansen/79/3/79 3 239/ pdf/-char/ en.

21. Brito KKG, Soares MJGO, Costa MML, Oliveira SHS. Practices and Limitations of Users with Hansen's Disease in Care of Skin Lesions J Nurs UFPE on line [Internet]. 2014 Jan [acesso em 3 out 2018]; 8(1). Disponível em: https://periodicos.ufpe.br/revistas/revistaenfermagem/article/viewFile/9600/9563.

22. Batista TVG, Vieira CSCA, Paula MAB de. A imagem corporal nas ações educativas em autocuidado para pessoas que tiveram hanseníase. Physis [Internet]. 2014 Mar [acesso em 03 out 2018]; 24(1). Disponível em: http://dx.doi.org/10.1590/S0103-73312014000100006.

23. Costa LG, Cortela D, Soares RC, Ignotti E. Factors associated with the worsening of the disability grade during leprosy treatment in Brazil. Leprosy Rev [Internet]. 2015 [acesso em 29 abr 2018]; 86(3). Disponivel em: https://www.researchgate.net/publication/288861864_Factors associated with the worsening of the disability grade during leprosy treatment in Brazil.

24. Pinheiro MGC, Miranda FAN, Simpson CA, Vitor AF, Lira ALBC. Limitações e incapacidades físicas no pós-alta em hanseníase: uma revisão integrativa. Rev baiana enferm [Internet]. 2016 [acesso em 29 abr 2018]; 30(2). Disponível em: http://doi.org/10.18471/rbe.v30i2.15669.

25. Galan NGA, Beluci ML, Marciano LHSC, Prado RBR, Oliveira NGG, Bonini AG, et al. Avaliação da prática do autocuidado domiciliar em hanseníase. Hansen Int [Internet]. 2014 [acesso em 07 maio 2018]; 39(2). Disponível em: http://www.ilsl.br/revista/detalhe artigo.php?id=12346.

26. Santana EMF, Brito KKG, Nogueira JA, Leadebal ODCP, Costa MML, Silva MA, et al. Deficiências e incapacidades na hanseníase: do diagnóstico à alta por cura. Rev Eletr Enf. [Internet]. 2018 [acesso em 18 maio 2018]; 20(15). Disponível em: http://doi.org/10.5216/ree.v20.50436.

27. Ministério da Saúde (BR). Secretaria de Vigilância em Saúde. Departamento de Vigilância Epidemiológica. Manual de Prevenção de Incapacidades [Internet]. Brasília: Ministério da Saúde; 2008 [acesso em 05 maio 2018]. Disponível em: http://bvsms.saude.gov.br/bvs/publicacoes/manual prevencao incapacidades.pdf.

28. Barbosa FPS, Mello IF, Pires JCB, Margarida TC, Silva Junior JRL, Santana CF. Incapacidades neurológicas provocadas pela hanseníase em uma unidade de saúde do município de Anápolis-GO, entre 2011 e 2013. Rev. Educ. Saúde [Internet]. 2016 [acesso em 07 maio 2018]; 4(2). Disponível em: http:// periodicos.unievangelica.edu.br/index.php/educacaoemsaude/article/view/2010.

29. Deshpande AV, Chaudhary SM, Mukhi JI, Bansode VW, Morey PD. Study of orofacial deformities in treated leprosy patients in central India. Int J Community Med Public Health [Internet]. 2017 [acesso em 01 out 2018]; 4(12). Disponível em: http://doi.org/10.18203/2394-6040.ijcmph20175349.

30. Araújo EJB, Araújo OD de, Araújo TME de, Almeida PD, Sena IV de O, Neri E de AR. Pós-alta de hanseníase: prevalência de incapacidades físicas e sobreposição de doenças. Cogitare enferm [Internet]. 2018 [acesso em 07 mar 2019]; 23 (4). Disponível em: http://dx.doi.org/10.5380/ce.v23i4.58080. 
Autor Correspondente:

Matheus de Medeiros Nóbrega

Universidade Federal da Paraíba

R. Maximiano Pedrosa, 103 - 58050460 - João Pessoa, PB, Brasil

E-mail: matheusnobrega0@gmail.com

Contribuição dos autores:

Contribuições substanciais para a concepção ou desenho do estudo; ou a aquisição, análise ou interpretação de dados do estudo - MMN, KKGB, EMVA, PSC, EMFS, MJGOS

Elaboração e revisão crítica do conteúdo intelectual do estudo - MMN, KKGB, MAS, MJGOS

Aprovação da versão final do estudo a ser publicado - MMN, KKGB, EMVA, PSC, EMFS, MAS, MJGOS

Responsável por todos os aspectos do estudo, assegurando as questões de precisão ou integridade de qualquer parte do estudo - MMN, KKGB 